\title{
Anticonvulsant Activity of a Combined Pharmacophore of Pyrazolo-pyridines with Lesser Toxicity in Mice
}

\author{
Nadeem Siddiqui, ${ }^{*}$ Waquar Ahsan, M Shamsher Alam, Ruhi Ali, Kamna Srivastava, ${ }^{\dagger}$ and Sharique Ahmed \\ Department of Pharmaceutical Chemistry, Faculty of Pharmacy, Hamdard University, Hamdard Nagar, \\ New Delhi-110062, India. *E-mail: nadeems_03@yahoo.co.in \\ ${ }^{\dagger}$ Center for Biomedical Research, University of Delhi, Delhi-110007, India \\ ${ }^{\ddagger}$ Department of Biochemistry, Faculty of Medicine, 7th October University, Misurata, Libya \\ Received October 25, 2010, Accepted December 7, 2010
}

\begin{abstract}
Various 2-amino-6-[3-(substituted phenyl)-5-phenyl-4,5-dihydropyrazol-1-yl]-4-(substituted phenyl)nicotinonitriles (3a-t) were designed and synthesized by clubbing two active anticonvulsant pharmacophores pyrazole and pyridine. All the synthesized compounds possessed the pharmacophoric elements essential for good anticonvulsant activity. The anticonvulsant screening was performed by maximal electroshock seizure (MES) and subcutaneous pentylenetetrazole (scPTZ) tests. Two compounds $3 \mathbf{i}$ and $\mathbf{3 s}$ showed significant anticonvulsant activity in both the screens with $\mathrm{ED}_{50}$ values $17.5 \mathrm{mg} / \mathrm{kg}$ and $22.6 \mathrm{mg} / \mathrm{kg}$ respectively in MES screen and $154.1 \mathrm{mg} / \mathrm{kg}$ and $242.6 \mathrm{mg} / \mathrm{kg}$ respectively in scPTZ screen. They were also found to have no acute toxic effects in mice when tested at elevated doses.
\end{abstract}

Key Words: Pyrazoles, Pyridines, Nicotinonitriles, Anticonvulsants, Neurotoxicity

\section{Introduction}

Recently pyrazole derivatives have constituted a vital part in the arsenal of anticonvulsant agents and its worth has been proved by the progressive findings in the literature. ${ }^{1-3}$ The anticonvulsant action of pyrazole moiety is ascribed to its unique properties of being a two-electron donor system and ability to act as a constrained pharmacophore at the receptor site.

A number of rather simple pyridine derivatives also exhibit high biological activity, nicotinic acid and its amide are contained in the organs of animals and are prosthetic group of enzymes codehydrogenase I and codehydrogenase II, which are hydrogen transfer agents and accomplish oxidation-reduction processes. Numerous currently used drugs in various diseases contain the pyridinyl pharmacophore that incited the scientists worldwide to investigate different derivatives possessing pyridine nucleus for anticonvulsant activity. The results obtained were found to be very encouraging ${ }^{4-7}$ and the research to get better agents is ongoing.

Our preceding research on pyrazole derivatives as anticonvulsant agents ${ }^{8}$ driven us to continue synthesizing newer derivatives and we intended to club pyridine nucleus to the pyrazole moiety. Clubbing the two active pharmacophores is expected to wield synergistic effects to the resulting molecule.

The suggested pharmacophore model for derivatives should have at least one aryl ring (R), one electron donor atom (D), and a second donor atom in close proximity to the NH group forming a hydrogen bond acceptor (HBA)/donor (HBD) unit. The titled compounds (3a-t) possessed all the required pharmacophoric elements (Fig. 1) as the phenyl ring attached to the pyrazole moiety can be referred to the aryl ring $(\mathrm{R})$, the imine of the pyrazole moiety can act as a two electron donor system (D) and the imine of the pyridine ring along with the $\mathrm{NH}$ of the free amino group constitute the hydrogen bond acceptor/donor unit (HAD).

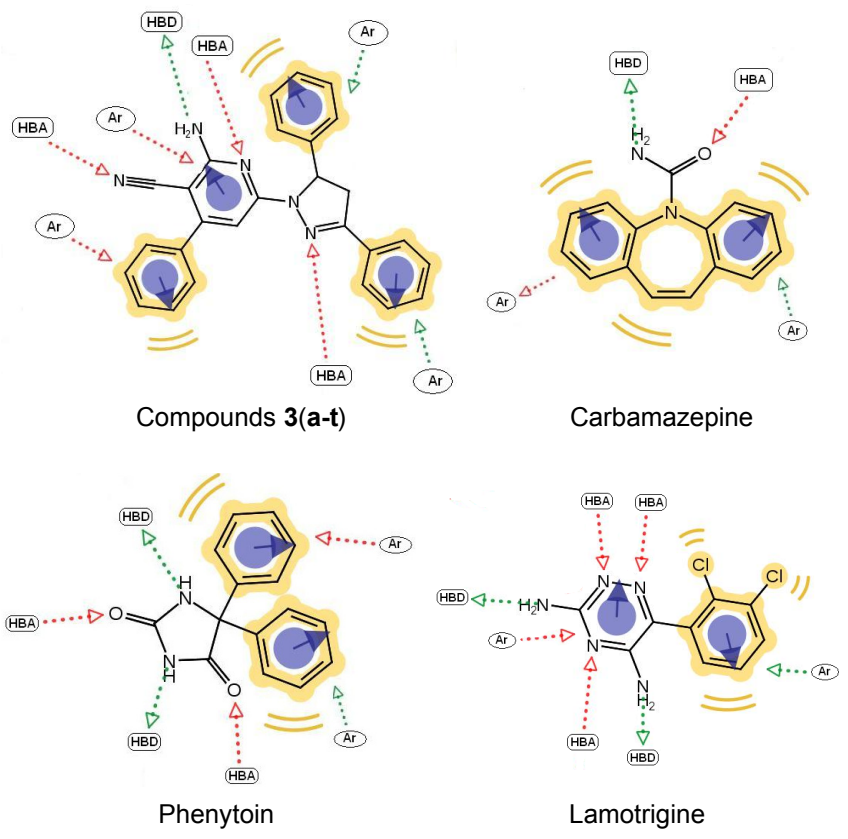

Figure 1. Anticonvulsant agents showing essential pharmacophoric elements present in their structure (HBA $=$ Hydrogen Bond Acceptor; HBD $=$ Hydrogen Bond Donor; Ar = Lipophilic Aryl Ring).

\section{Experimental}

Chemistry. The melting points were determined in open capillary tubes in a Hicon melting point apparatus and are uncorrected. The homogen elemental analyses $(\mathrm{C}, \mathrm{H}, \mathrm{N})$ of all compounds were performed on the CHNS Elimentar (Analysen systime, GmbH) Germany Vario EL III. All the Fourier transform infra red (FT-IR) spectra were recorded in $\mathrm{KBr}$ pellets on a Jasco FT/IR 410 spectrometer. The ${ }^{1} \mathrm{H}$ NMR spectra were recorded on a Bruker 400 Ultra shield $^{\mathrm{TM}}(400 \mathrm{MHz}) \mathrm{NMR}$ 
spectrometer. Chemical shifts $(\delta)$ are expressed in ppm relative to tetramethylsilane (TMS) as an internal standard. The homogeneity of the compounds was checked by thin layer chromatography (TLC) on silica gel G (Merck) coated plates by using toluene:ethylacetate:formic acid $(5: 4: 1)$ as solvent system. Iodine chamber and UV lamp were used for the visualization of TLC spots.

General Procedure for the Synthesis of Titled Compounds (3a-t).

1-(Substituted phenyl)-3-phenylpropenones (1a-d): Substituted acetophenones $(0.43 \mathrm{~mol})$ and benzaldehyde $(0.43 \mathrm{~mol})$ were added to the solution of $40 \% \mathrm{NaOH}(200 \mathrm{~mL})$ and rectified spirit $(99.8 \%, 12.5 \mathrm{~mL})$ at $5-10{ }^{\circ} \mathrm{C}$ and stirred for $4 \mathrm{~h}$, maintaining the temperature $25^{\circ} \mathrm{C}$. The solid mass thus obtained was kept on the ice chest over night, washed with water, dried and recrystallized using ethanol to afford the target compounds (1a-d).

1-[3-(Substituted phenyl)-5-phenyl-4,5-dihydropyrazol-1yl]ethanones (2a-d): A solution of the corresponding chalcones (1a-d, $0.002 \mathrm{~mol})$, hydrazine hydrate $(0.006 \mathrm{~mol})$ and $10 \mathrm{~mL}$ of acetic acid was refluxed for $5 \mathrm{~h}$. Subsequently, catalytic amount of dilute hydrochloric acid ( 4 - 5 drops) was added and refluxed for $30 \mathrm{~min}$. After cooling $30 \mathrm{~mL}$ water was added and the resulting precipitate was filtered, washed with water.'

2-Amino-6-[3-(substituted phenyl)-5-phenyl-4,5-dihydropyrazol-1-yl]-4-(substituted phenyl)nicotinonitriles (3a-t): To the solution of starting material (2a-d, $0.01 \mathrm{~mol})$ in ethanol (40 $\mathrm{mL})$, added appropriate aromatic aldehydes $(0.01$ mole $)$. This mixture was treated with malononitrile $(0.01$ mole, $0.66 \mathrm{~g})$ and ammonium acetate $(2 \mathrm{~g})$ and then refluxed for $10 \mathrm{~h}$, a crystalline precipitate get separated (Hantzsch Pyridine Synthesis). The precipitate was filtered, washed with ethanol, dried and recrystallized from acetic acid:ethanol.

2-Amino-6-(3,5-diphenyl-4,5-dihydropyrazol-1-yl)-4-(4hydroxyphenyl)nicotinonitrile (3a): Yield 73\%, mp $211^{\circ} \mathrm{C}$. IR $(\mathrm{KBr}) \mathrm{cm}^{-1}: 3715(\mathrm{OH}), 3422\left(\mathrm{NH}_{2}\right), 3049\left(\mathrm{Ar}-\mathrm{CH}_{\text {str }}\right), 1224$ $\left(\mathrm{CN}_{\mathrm{str}}\right) ;{ }^{1} \mathrm{H}$ NMR $\left(\mathrm{CDCl}_{3}\right) \delta 3.16-3.22\left(\mathrm{dd}, 1 \mathrm{H}, \mathrm{H}_{\mathrm{A}}, J_{\mathrm{AB}}=4.8\right.$ $\left.\mathrm{Hz}, J_{\mathrm{AX}}=4.8 \mathrm{~Hz}\right), 3.74-3.81\left(\mathrm{dd}, 1 \mathrm{H}, \mathrm{H}_{\mathrm{B}}, J_{\mathrm{AB}}=11.6 \mathrm{~Hz}, J_{\mathrm{BX}}=\right.$ $11.6 \mathrm{~Hz}), 5.57-5.61\left(\mathrm{dd}, 1 \mathrm{H}, \mathrm{H}_{\mathrm{X}}, J_{\mathrm{AX}}=4.8 \mathrm{~Hz}, J_{\mathrm{BX}}=4.8 \mathrm{~Hz}\right)$, $6.69\left(\mathrm{~d}, 2 \mathrm{H}, \mathrm{NH}_{2}, J=8.8 \mathrm{~Hz}, \mathrm{D}_{2} \mathrm{O}\right.$ exchangeable), 7.21-7.75 $(\mathrm{m}, 15 \mathrm{H}, \mathrm{ArH}), 9.48\left(\mathrm{~s}, 1 \mathrm{H}, \mathrm{OH}, \mathrm{D}_{2} \mathrm{O}\right.$ exchangeable $)$.

2-Amino-6-(3,5-diphenyl-4,5-dihydropyrazol-1-yl)-4-(2hydroxyphenyl)nicotinonitrile (3b): Yield $81 \%$, mp $202{ }^{\circ} \mathrm{C}$. IR (KBr) cm ${ }^{-1}$ : $3678(\mathrm{OH}), 3416\left(\mathrm{NH}_{2}\right), 2976\left(\mathrm{Ar}-\mathrm{CH}_{\mathrm{str}}\right)$, $1206\left(\mathrm{CN}_{\mathrm{str}}\right) ;{ }^{1} \mathrm{H} \mathrm{NMR}\left(\mathrm{CDCl}_{3}\right) \delta 3.15-3.24\left(\mathrm{dd}, 1 \mathrm{H}, \mathrm{H}_{\mathrm{A}}, J_{\mathrm{AB}}=\right.$ $\left.4.8 \mathrm{~Hz}, J_{\mathrm{AX}}=4.8 \mathrm{~Hz}\right), 3.71-3.85\left(\mathrm{dd}, 1 \mathrm{H}, \mathrm{H}_{\mathrm{B}}, J_{\mathrm{AB}}=11.6 \mathrm{~Hz}\right.$, $\left.J_{\mathrm{BX}}=11.6 \mathrm{~Hz}\right), 5.54-5.67\left(\mathrm{dd}, 1 \mathrm{H}, \mathrm{H}_{\mathrm{X}}, J_{\mathrm{AX}}=4.8 \mathrm{~Hz}, J_{\mathrm{BX}}=4.8\right.$ $\mathrm{Hz}), 6.65\left(\mathrm{~d}, 2 \mathrm{H}, \mathrm{NH}_{2}, J=8.6 \mathrm{~Hz}, \mathrm{D}_{2} \mathrm{O}\right.$ exchangeable), 7.16-7.76 $(\mathrm{m}, 15 \mathrm{H}, \mathrm{ArH}), 9.51$ (s, 1H, OH, $\mathrm{D}_{2} \mathrm{O}$ exchangeable).

2-Amino-6-(3,5-diphenyl-4,5-dihydropyrazol-1-yl)-4-(4methylphenyl)nicotinonitrile (3c): Yield 59\%, mp $188^{\circ} \mathrm{C}$. IR $(\mathrm{KBr}) \mathrm{cm}^{-1}$ : $3446\left(\mathrm{NH}_{2}\right), 2978\left(\mathrm{Ar}-\mathrm{CH}_{\text {str }}\right), 1222\left(\mathrm{CN}_{\mathrm{str}}\right) ;{ }^{1} \mathrm{H}$ $\mathrm{NMR}\left(\mathrm{CDCl}_{3}\right) \delta 2.19\left(\mathrm{~s}, 3 \mathrm{H}, \mathrm{CH}_{3}\right), 3.11-3.20\left(\mathrm{dd}, 1 \mathrm{H}, \mathrm{H}_{\mathrm{A}}, J_{\mathrm{AB}}=\right.$ $\left.4.6 \mathrm{~Hz}, J_{\mathrm{AX}}=4.6 \mathrm{~Hz}\right), 3.68-3.81\left(\mathrm{dd}, 1 \mathrm{H}, \mathrm{H}_{\mathrm{B}}, J_{\mathrm{AB}}=10.4 \mathrm{~Hz}\right.$, $\left.J_{\mathrm{BX}}=10.4 \mathrm{~Hz}\right), 5.52-5.65\left(\mathrm{dd}, 1 \mathrm{H}, \mathrm{H}_{\mathrm{X}}, J_{\mathrm{AX}}=4.6 \mathrm{~Hz}, J_{\mathrm{BX}}=4.6\right.$ $\mathrm{Hz}), 6.69$ (d, 2H, $\mathrm{NH}_{2}, J=8.8 \mathrm{~Hz}, \mathrm{D}_{2} \mathrm{O}$ exchangeable), 7.21-7.81 $(\mathrm{m}, 15 \mathrm{H}, \mathrm{ArH})$.

2-Amino-6-(3,5-diphenyl-4,5-dihydropyrazol-1-yl)-4-(4- methoxyphenyl)nicotinonitrile (3d): Yield $72 \%, \operatorname{mp~} 197{ }^{\circ} \mathrm{C}$. IR $(\mathrm{KBr}) \mathrm{cm}^{-1}: 3415\left(\mathrm{NH}_{2}\right), 3001\left(\mathrm{Ar}-\mathrm{CH}_{\text {str }}\right), 1178\left(\mathrm{CN}_{\text {str }}\right) ;{ }^{1} \mathrm{H}$ NMR $\left(\mathrm{CDCl}_{3}\right) \delta 3.41\left(\mathrm{~s}, 3 \mathrm{H}, \mathrm{OCH}_{3}\right), 3.14-3.22\left(\mathrm{dd}, 1 \mathrm{H}, \mathrm{H}_{\mathrm{A}}\right.$, $\left.J_{\mathrm{AB}}=5.2 \mathrm{~Hz}, J_{\mathrm{AX}}=5.2 \mathrm{~Hz}\right), 3.65-3.79\left(\mathrm{dd}, 1 \mathrm{H}, \mathrm{H}_{\mathrm{B}}, J_{\mathrm{AB}}=11.8\right.$ $\left.\mathrm{Hz}, J_{\mathrm{BX}}=11.8 \mathrm{~Hz}\right), 5.50-5.65\left(\mathrm{dd}, 1 \mathrm{H}, \mathrm{H}_{\mathrm{X}}, J_{\mathrm{AX}}=4.6 \mathrm{~Hz}, J_{\mathrm{BX}}=\right.$ $4.6 \mathrm{~Hz}), 6.69$ (d, 2H, $\mathrm{NH}_{2}, J=8.4 \mathrm{~Hz}, \mathrm{D}_{2} \mathrm{O}$ exchangeable), 7.18-7.86 (m, 15H, ArH).

2-Amino-6-(3,5-diphenyl-4,5-dihydropyrazol-1-yl)-4-phenylnicotinonitrile (3e): Yield 54\%, mp $206^{\circ} \mathrm{C}$. IR (KBr) $\mathrm{cm}^{-1}$ : $3478\left(\mathrm{NH}_{2}\right), 3064\left(\mathrm{Ar}-\mathrm{CH}_{\mathrm{str}}\right), 1204\left(\mathrm{CN}_{\mathrm{str}}\right) ;{ }^{1} \mathrm{H} \mathrm{NMR}\left(\mathrm{CDCl}_{3}\right)$ $\delta 3.12-3.20\left(\mathrm{dd}, 1 \mathrm{H}, \mathrm{H}_{\mathrm{A}}, J_{\mathrm{AB}}=4.6 \mathrm{~Hz}, J_{\mathrm{AX}}=4.6 \mathrm{~Hz}\right), 3.63-3.74$ $\left(\mathrm{dd}, 1 \mathrm{H}, \mathrm{H}_{\mathrm{B}}, J_{\mathrm{AB}}=11.6 \mathrm{~Hz}, J_{\mathrm{BX}}=11.6 \mathrm{~Hz}\right), 5.53-5.64(\mathrm{dd}, 1 \mathrm{H}$, $\left.\mathrm{H}_{\mathrm{X}}, J_{\mathrm{AX}}=4.8 \mathrm{~Hz}, J_{\mathrm{BX}}=4.8 \mathrm{~Hz}\right), 6.61\left(\mathrm{~d}, 2 \mathrm{H}, \mathrm{NH}_{2}, J=8.6 \mathrm{~Hz}\right.$, $\mathrm{D}_{2} \mathrm{O}$ exchangeable), 7.12-7.89 (m, 16H, ArH).

2-Amino-6-[3-(4-bromophenyl)-5-phenyl-4,5-dihydropyrazol-1-yl]-4-(4-hydroxyphenyl) nicotinonitrile (3f): Yield 69\%, $\mathrm{mp} 269^{\circ} \mathrm{C}$. IR $(\mathrm{KBr}) \mathrm{cm}^{-1}$ : $3615(\mathrm{OH}), 3456\left(\mathrm{NH}_{2}\right), 3015$ (Ar$\left.\mathrm{CH}_{\mathrm{str}}\right), 1212\left(\mathrm{CN}_{\mathrm{str}}\right) ;{ }^{1} \mathrm{H} \mathrm{NMR}\left(\mathrm{CDCl}_{3}\right) \delta 3.09-3.18(\mathrm{dd}, 1 \mathrm{H}$, $\left.\mathrm{H}_{\mathrm{A}}, J_{\mathrm{AB}}=4.4 \mathrm{~Hz}, J_{\mathrm{AX}}=4.4 \mathrm{~Hz}\right), 3.61-3.79\left(\mathrm{dd}, 1 \mathrm{H}, \mathrm{H}_{\mathrm{B}}, J_{\mathrm{AB}}=\right.$ $\left.12.2 \mathrm{~Hz}, J_{\mathrm{BX}}=12.2 \mathrm{~Hz}\right), 5.51-5.66\left(\mathrm{dd}, 1 \mathrm{H}, \mathrm{H}_{\mathrm{X}}, J_{\mathrm{AX}}=4.6 \mathrm{~Hz}\right.$, $\left.J_{\mathrm{BX}}=4.6 \mathrm{~Hz}\right), 6.69\left(\mathrm{~d}, 2 \mathrm{H}, \mathrm{NH}_{2}, J=8.4 \mathrm{~Hz}, \mathrm{D}_{2} \mathrm{O}\right.$ exchangeable), 7.15-7.81 (m, 14H, ArH), 9.39 (s, 1H, OH, $\mathrm{D}_{2} \mathrm{O}$ exchangeable).

2-Amino-6-[3-(4-bromophenyl)-5-phenyl-4,5-dihydropyrazol-1-yl]-4-(2-hydroxyphenyl) nicotinonitrile (3g): Yield 77\%, $\mathrm{mp} 257^{\circ} \mathrm{C}$. IR $(\mathrm{KBr}) \mathrm{cm}^{-1}$ : $3645(\mathrm{OH}), 3412\left(\mathrm{NH}_{2}\right), 3054(\mathrm{Ar}-$ $\left.\mathrm{CH}_{\mathrm{str}}\right), 1249\left(\mathrm{CN}_{\mathrm{str}}\right) ;{ }^{1} \mathrm{H} \mathrm{NMR}\left(\mathrm{CDCl}_{3}\right) \delta 3.11-3.19(\mathrm{dd}, 1 \mathrm{H}$, $\left.\mathrm{H}_{\mathrm{A}}, J_{\mathrm{AB}}=4.8 \mathrm{~Hz}, J_{\mathrm{AX}}=4.8 \mathrm{~Hz}\right), 3.60-3.81\left(\mathrm{dd}, 1 \mathrm{H}, \mathrm{H}_{\mathrm{B}}, J_{\mathrm{AB}}=\right.$ $\left.11.4 \mathrm{~Hz}, J_{\mathrm{BX}}=11.4 \mathrm{~Hz}\right), 5.12-5.69\left(\mathrm{dd}, 1 \mathrm{H}, \mathrm{H}_{\mathrm{X}}, J_{\mathrm{AX}}=4.8 \mathrm{~Hz}\right.$, $\left.J_{\mathrm{BX}}=4.8 \mathrm{~Hz}\right), 6.61\left(\mathrm{~d}, 2 \mathrm{H}, \mathrm{NH}_{2}, J=8.6 \mathrm{~Hz}, \mathrm{D}_{2} \mathrm{O}\right.$ exchangeable), 7.11-7.78 (m, 14H, ArH), 9.45 (s, 1H, OH, $\mathrm{D}_{2} \mathrm{O}$ exchangeable).

2-Amino-6-[3-(4-bromophenyl)-5-phenyl-4,5-dihydropyrazol-1-yl]-4-(4-methylphenyl) nicotinonitrile (3h): Yield 67\%, $\mathrm{mp} 181{ }^{\circ} \mathrm{C}$. IR $(\mathrm{KBr}) \mathrm{cm}^{-1}: 3415\left(\mathrm{NH}_{2}\right), 2997\left(\mathrm{Ar}-\mathrm{CH}_{\text {str }}\right), 1256$ $\left(\mathrm{CN}_{\mathrm{str}}\right) ;{ }^{1} \mathrm{H} \mathrm{NMR}\left(\mathrm{CDCl}_{3}\right) \delta 2.31\left(\mathrm{~s}, 3 \mathrm{H}, \mathrm{CH}_{3}\right), 3.14-3.26(\mathrm{dd}$, $\left.1 \mathrm{H}, \mathrm{H}_{\mathrm{A}}, J_{\mathrm{AB}}=4.6 \mathrm{~Hz}, J_{\mathrm{AX}}=4.6 \mathrm{~Hz}\right), 3.61-3.84\left(\mathrm{dd}, 1 \mathrm{H}, \mathrm{H}_{\mathrm{B}}\right.$, $\left.J_{\mathrm{AB}}=11.4 \mathrm{~Hz}, J_{\mathrm{BX}}=11.4 \mathrm{~Hz}\right), 5.54-5.69\left(\mathrm{dd}, 1 \mathrm{H}, \mathrm{H}_{\mathrm{X}}, J_{\mathrm{AX}}=\right.$ $\left.4.6 \mathrm{~Hz}, J_{\mathrm{BX}}=4.6 \mathrm{~Hz}\right), 6.63\left(\mathrm{~d}, 2 \mathrm{H}, \mathrm{NH}_{2}, J=8.8 \mathrm{~Hz}, \mathrm{D}_{2} \mathrm{O}\right.$ exchangeable), 7.21-7.80 (m, 14H, ArH).

2-Amino-6-[3-(4-bromophenyl)-5-phenyl-4,5-dihydropyrazol-1-yl]-4-(4-methoxyphenyl) nicotinonitrile (3i): Yield 58\%, $\mathrm{mp} 174{ }^{\circ} \mathrm{C}$. IR $(\mathrm{KBr}) \mathrm{cm}^{-1}$ : $3449\left(\mathrm{NH}_{2}\right), 2982\left(\mathrm{Ar}-\mathrm{CH}_{\mathrm{str}}\right), 1278$ $\left(\mathrm{CN}_{\mathrm{str}}\right) ;{ }^{1} \mathrm{H} \mathrm{NMR}\left(\mathrm{CDCl}_{3}\right) \delta 3.56\left(\mathrm{~s}, 3 \mathrm{H}, \mathrm{OCH}_{3}\right), 3.10-3.24(\mathrm{dd}$, $\left.1 \mathrm{H}, \mathrm{H}_{\mathrm{A}}, J_{\mathrm{AB}}=4.2 \mathrm{~Hz}, J_{\mathrm{AX}}=4.2 \mathrm{~Hz}\right), 3.61-3.76\left(\mathrm{dd}, 1 \mathrm{H}, \mathrm{H}_{\mathrm{B}}\right.$, $\left.J_{\mathrm{AB}}=11.2 \mathrm{~Hz}, J_{\mathrm{BX}}=11.2 \mathrm{~Hz}\right), 5.53-5.67\left(\mathrm{dd}, 1 \mathrm{H}, \mathrm{H}_{\mathrm{X}}, J_{\mathrm{AX}}=\right.$ $\left.4.2 \mathrm{~Hz}, J_{\mathrm{BX}}=4.2 \mathrm{~Hz}\right), 6.66\left(\mathrm{~d}, 2 \mathrm{H}, \mathrm{NH}_{2}, J=8.4 \mathrm{~Hz}, \mathrm{D}_{2} \mathrm{O}\right.$ exchangeable), 7.14-7.82 (m, 14H, ArH).

2-Amino-6-[3-(4-bromophenyl)-5-phenyl-4,5-dihydropyrazol-1-yl]-4-phenylnicotinonitrile (3j): Yield 79\%, mp $247^{\circ} \mathrm{C}$. IR $(\mathrm{KBr}) \mathrm{cm}^{-1}$ : $3416\left(\mathrm{NH}_{2}\right), 3019\left(\mathrm{Ar}-\mathrm{CH}_{\text {str }}\right), 1249\left(\mathrm{CN}_{\mathrm{str}}\right) ;{ }^{1} \mathrm{H}$ $\operatorname{NMR}\left(\mathrm{CDCl}_{3}\right) \delta 3.14-3.27\left(\mathrm{dd}, 1 \mathrm{H}, \mathrm{H}_{\mathrm{A}}, J_{\mathrm{AB}}=4.8 \mathrm{~Hz}, J_{\mathrm{AX}}=4.8\right.$ $\mathrm{Hz}), 3.61-3.76\left(\mathrm{dd}, 1 \mathrm{H}, \mathrm{H}_{\mathrm{B}}, J_{\mathrm{AB}}=11.4 \mathrm{~Hz}, J_{\mathrm{BX}}=11.4 \mathrm{~Hz}\right)$, $5.52-5.66\left(\mathrm{dd}, 1 \mathrm{H}, \mathrm{H}_{\mathrm{X}}, J_{\mathrm{AX}}=4.6 \mathrm{~Hz}, J_{\mathrm{BX}}=4.6 \mathrm{~Hz}\right), 6.64(\mathrm{~d}, 2 \mathrm{H}$, $\mathrm{NH}_{2}, J=8.6 \mathrm{~Hz}, \mathrm{D}_{2} \mathrm{O}$ exchangeable), 7.14-7.83 (m, 15H, ArH).

2-Amino-6-[3-(4-chlorophenyl)-5-phenyl-4,5-dihydropyrazol-1-yl]-4-(4-hydroxyphenyl) nicotinonitrile (3k): Yield 74\%, $\mathrm{mp} 216^{\circ} \mathrm{C}$. IR $(\mathrm{KBr}) \mathrm{cm}^{-1}: 3645(\mathrm{OH}), 3416\left(\mathrm{NH}_{2}\right), 3046(\mathrm{Ar}-$ $\left.\mathrm{CH}_{\text {str }}\right), 1236\left(\mathrm{CN}_{\mathrm{str}}\right) ;{ }^{1} \mathrm{H} \mathrm{NMR}\left(\mathrm{CDCl}_{3}\right) \delta 3.12-3.24(\mathrm{dd}, 1 \mathrm{H}$, $\left.\mathrm{H}_{\mathrm{A}}, J_{\mathrm{AB}}=4.6 \mathrm{~Hz}, J_{\mathrm{AX}}=4.6 \mathrm{~Hz}\right), 3.64-3.76\left(\mathrm{dd}, 1 \mathrm{H}, \mathrm{H}_{\mathrm{B}}, J_{\mathrm{AB}}=\right.$ 
$\left.12.4 \mathrm{~Hz}, J_{\mathrm{BX}}=12.4 \mathrm{~Hz}\right), 5.53-5.69\left(\mathrm{dd}, 1 \mathrm{H}, \mathrm{H}_{\mathrm{X}}, J_{\mathrm{AX}}=4.8 \mathrm{~Hz}\right.$, $\left.J_{\mathrm{BX}}=4.8 \mathrm{~Hz}\right), 6.63\left(\mathrm{~d}, 2 \mathrm{H}, \mathrm{NH}_{2}, J=8.8 \mathrm{~Hz}, \mathrm{D}_{2} \mathrm{O}\right.$ exchangeable), 7.12-7.80 (m, 14H, ArH), 9.41 (s, 1H, OH, $\mathrm{D}_{2} \mathrm{O}$ exchangeable).

2-Amino-6-[3-(4-chlorophenyl)-5-phenyl-4,5-dihydropyrazol-1-yl]-4-(2-hydroxyphenyl) nicotinonitrile (3I): Yield 63\%, $\mathrm{mp} 249^{\circ} \mathrm{C}$. IR (KBr) cm ${ }^{-1}: 3678(\mathrm{OH}), 3449\left(\mathrm{NH}_{2}\right), 3057$ (Ar$\left.\mathrm{CH}_{\text {str }}\right), 1234\left(\mathrm{CN}_{\text {str }}\right) ;{ }^{1} \mathrm{H}$ NMR $\left(\mathrm{CDCl}_{3}\right) \delta 3.10-3.26(\mathrm{dd}, 1 \mathrm{H}$, $\left.\mathrm{H}_{\mathrm{A}}, J_{\mathrm{AB}}=4.8 \mathrm{~Hz}, J_{\mathrm{AX}}=4.8 \mathrm{~Hz}\right), 3.61-3.76\left(\mathrm{dd}, 1 \mathrm{H}, \mathrm{H}_{\mathrm{B}}, J_{\mathrm{AB}}=\right.$ $\left.11.8 \mathrm{~Hz}, J_{\mathrm{BX}}=11.8 \mathrm{~Hz}\right), 5.51-5.68\left(\mathrm{dd}, 1 \mathrm{H}, \mathrm{H}_{\mathrm{X}}, J_{\mathrm{AX}}=4.8 \mathrm{~Hz}\right.$, $\left.J_{\mathrm{BX}}=4.8 \mathrm{~Hz}\right), 6.65\left(\mathrm{~d}, 2 \mathrm{H}, \mathrm{NH}_{2}, J=8.6 \mathrm{~Hz}, \mathrm{D}_{2} \mathrm{O}\right.$ exchangeable), 7.10-7.79 (m, 14H, ArH), 9.49 ( $\mathrm{s}, 1 \mathrm{H}, \mathrm{OH}, \mathrm{D}_{2} \mathrm{O}$ exchangeable).

2-Amino-6-[3-(4-chlorophenyl)-5-phenyl-4,5-dihydropyrazol-1-yl]-4(4-methylphenyl) nicotinonitrile (3m): Yield 76\%, mp $194{ }^{\circ} \mathrm{C}$. IR $(\mathrm{KBr}) \mathrm{cm}^{-1}$ : $3416\left(\mathrm{NH}_{2}\right), 2896\left(\mathrm{Ar}-\mathrm{CH}_{\mathrm{str}}\right), 1203$ $\left(\mathrm{CN}_{\mathrm{str}}\right) ;{ }^{1} \mathrm{H} \mathrm{NMR}\left(\mathrm{CDCl}_{3}\right) \delta 2.35\left(\mathrm{~s}, 3 \mathrm{H}, \mathrm{CH}_{3}\right), 3.19-3.31(\mathrm{dd}$, $\left.1 \mathrm{H}, \mathrm{H}_{\mathrm{A}}, J_{\mathrm{AB}}=5.6 \mathrm{~Hz}, J_{\mathrm{AX}}=5.6 \mathrm{~Hz}\right), 3.68-3.87\left(\mathrm{dd}, 1 \mathrm{H}, \mathrm{H}_{\mathrm{B}}\right.$, $\left.J_{\mathrm{AB}}=11.8 \mathrm{~Hz}, J_{\mathrm{BX}}=11.8 \mathrm{~Hz}\right), 5.53-5.68\left(\mathrm{dd}, 1 \mathrm{H}, \mathrm{H}_{\mathrm{X}}, J_{\mathrm{AX}}=\right.$ $\left.4.6 \mathrm{~Hz}, J_{\mathrm{BX}}=4.6 \mathrm{~Hz}\right), 6.68\left(\mathrm{~d}, 2 \mathrm{H}, \mathrm{NH}_{2}, J=8.6 \mathrm{~Hz}, \mathrm{D}_{2} \mathrm{O}\right.$ exchangeable), 7.26-7.87 (m, 14H, ArH).

2-Amino-6-[3-(4-chlorophenyl)-5-phenyl-4,5-dihydropyrazol-1-yl]-4-(4-methoxyphenyl) nicotinonitrile (3n): Yield 55\%, mp $209^{\circ} \mathrm{C}$. IR ( $\left.\mathrm{KBr}\right) \mathrm{cm}^{-1}: 3476\left(\mathrm{NH}_{2}\right), 2998\left(\mathrm{Ar}-\mathrm{CH}_{\text {str }}\right), 1256$ $\left(\mathrm{CN}_{\mathrm{str}}\right) ;{ }^{1} \mathrm{H} \mathrm{NMR}\left(\mathrm{CDCl}_{3}\right) \delta 3.64\left(\mathrm{~s}, 3 \mathrm{H}, \mathrm{OCH}_{3}\right), 3.13-3.27$ (dd, $\left.1 \mathrm{H}, \mathrm{H}_{\mathrm{A}}, J_{\mathrm{AB}}=4.4 \mathrm{~Hz}, J_{\mathrm{AX}}=4.4 \mathrm{~Hz}\right), 3.60-3.76\left(\mathrm{dd}, 1 \mathrm{H}, \mathrm{H}_{\mathrm{B}}\right.$, $\left.J_{\mathrm{AB}}=11.4 \mathrm{~Hz}, J_{\mathrm{BX}}=11.4 \mathrm{~Hz}\right), 5.56-5.69\left(\mathrm{dd}, 1 \mathrm{H}, \mathrm{H}_{\mathrm{X}}, J_{\mathrm{AX}}=\right.$ $\left.4.6 \mathrm{~Hz}, J_{\mathrm{BX}}=4.6 \mathrm{~Hz}\right), 6.68\left(\mathrm{~d}, 2 \mathrm{H}, \mathrm{NH}_{2}, J=8.6 \mathrm{~Hz}, \mathrm{D}_{2} \mathrm{O}\right.$ exchangeable), 7.13-7.80 (m, 14H, ArH).

2-Amino-6-[3-(4-chlorophenyl)-5-phenyl-4,5-dihydropyrazol-1-yl]-4-phenylnicotinonitrile (3o): Yield 67\%, mp $248{ }^{\circ} \mathrm{C}$. IR $(\mathrm{KBr}) \mathrm{cm}^{-1}: 3454\left(\mathrm{NH}_{2}\right), 3043\left(\mathrm{Ar}-\mathrm{CH}_{\text {str }}\right), 1279\left(\mathrm{CN}_{\mathrm{str}}\right) ;{ }^{1} \mathrm{H}$ $\operatorname{NMR}\left(\mathrm{CDCl}_{3}\right) \delta 3.15-3.26\left(\mathrm{dd}, 1 \mathrm{H}, \mathrm{H}_{\mathrm{A}}, J_{\mathrm{AB}}=4.6 \mathrm{~Hz}, J_{\mathrm{AX}}=4.6\right.$ $\mathrm{Hz}), 3.63-3.77\left(\mathrm{dd}, 1 \mathrm{H}, \mathrm{H}_{\mathrm{B}}, J_{\mathrm{AB}}=11.6 \mathrm{~Hz}, J_{\mathrm{BX}}=11.6 \mathrm{~Hz}\right)$, 5.54-5.69 (dd, $\left.1 \mathrm{H}, \mathrm{H}_{\mathrm{X}}, J_{\mathrm{AX}}=4.8 \mathrm{~Hz}, J_{\mathrm{BX}}=4.8 \mathrm{~Hz}\right), 6.66(\mathrm{~d}$, $2 \mathrm{H}, \mathrm{NH}_{2}, J=8.4 \mathrm{~Hz}, \mathrm{D}_{2} \mathrm{O}$ exchangeable), 7.11-7.79 (m, $15 \mathrm{H}$, ArH).

2-Amino-4-(4-hydroxyphenyl)-6-[3-(4-nitrophenyl)-5-phenyl-4,5-dihydropyrazol-1-yl]nicotinonitrile (3p): Yield 71\%, $\mathrm{mp} 234^{\circ} \mathrm{C}$. IR (KBr) cm ${ }^{-1}$ : $3616(\mathrm{OH}), 3421\left(\mathrm{NH}_{2}\right), 3024(\mathrm{Ar}-$ $\left.\mathrm{CH}_{\text {str }}\right), 1289\left(\mathrm{CN}_{\text {str }}\right) ;{ }^{1} \mathrm{H} \mathrm{NMR}\left(\mathrm{CDCl}_{3}\right) \delta 3.15-3.29(\mathrm{dd}, 1 \mathrm{H}$, $\left.\mathrm{H}_{\mathrm{A}}, J_{\mathrm{AB}}=4.8 \mathrm{~Hz}, J_{\mathrm{AX}}=4.8 \mathrm{~Hz}\right), 3.61-3.75\left(\mathrm{dd}, 1 \mathrm{H}, \mathrm{H}_{\mathrm{B}}, J_{\mathrm{AB}}=\right.$ $\left.11.4 \mathrm{~Hz}, J_{\mathrm{BX}}=11.4 \mathrm{~Hz}\right), 5.51-5.67\left(\mathrm{dd}, 1 \mathrm{H}, \mathrm{H}_{\mathrm{X}}, J_{\mathrm{AX}}=4.8 \mathrm{~Hz}\right.$, $\left.J_{\mathrm{BX}}=4.8 \mathrm{~Hz}\right), 6.67\left(\mathrm{~d}, 2 \mathrm{H}, \mathrm{NH}_{2}, J=8.8 \mathrm{~Hz}, \mathrm{D}_{2} \mathrm{O}\right.$ exchangeable), 7.14-7.79 (m, 14H, ArH), $9.46\left(\mathrm{~s}, 1 \mathrm{H}, \mathrm{OH}, \mathrm{D}_{2} \mathrm{O}\right.$ exchangeable).

2-Amino-4-(2-hydroxyphenyl)-6-[3-(4-nitrophenyl)-5-phenyl-4,5-dihydropyrazol-1-yl]nicotinonitrile (3q): Yield 57\%, $\mathrm{mp} 214{ }^{\circ} \mathrm{C}$. IR (KBr) cm ${ }^{-1}: 3654(\mathrm{OH}), 3454\left(\mathrm{NH}_{2}\right), 3007$ (Ar$\left.\mathrm{CH}_{\mathrm{str}}\right), 1251\left(\mathrm{CN}_{\mathrm{str}}\right) ;{ }^{1} \mathrm{H} \mathrm{NMR}\left(\mathrm{CDCl}_{3}\right) \delta 3.14-3.29(\mathrm{dd}, 1 \mathrm{H}$, $\left.\mathrm{H}_{\mathrm{A}}, J_{\mathrm{AB}}=4.6 \mathrm{~Hz}, J_{\mathrm{AX}}=4.6 \mathrm{~Hz}\right), 3.63-3.77\left(\mathrm{dd}, 1 \mathrm{H}, \mathrm{H}_{\mathrm{B}}, J_{\mathrm{AB}}=\right.$ $\left.11.4 \mathrm{~Hz}, J_{\mathrm{BX}}=11.4 \mathrm{~Hz}\right), 5.53-5.67\left(\mathrm{dd}, 1 \mathrm{H}, \mathrm{H}_{\mathrm{X}}, J_{\mathrm{AX}}=4.6 \mathrm{~Hz}\right.$, $\left.J_{\mathrm{BX}}=4.6 \mathrm{~Hz}\right), 6.69\left(\mathrm{~d}, 2 \mathrm{H}, \mathrm{NH}_{2}, J=8.6 \mathrm{~Hz}, \mathrm{D}_{2} \mathrm{O}\right.$ exchangeable), 7.11-7.76 (m, 14H, ArH), 9.51 (s, 1H, OH, $\mathrm{D}_{2} \mathrm{O}$ exchangeable).

2-Amino-4-(4-methylphenyl)-6-[3-(4-nitrophenyl)-5-phenyl-4,5-dihydropyrazol-1-yl] nicotinonitrile (3r): Yield 54\%, mp $179^{\circ} \mathrm{C}$. IR (KBr) cm ${ }^{-1}$ : $3457\left(\mathrm{NH}_{2}\right), 3016\left(\mathrm{Ar}-\mathrm{CH}_{\text {str }}\right), 1252$ $\left(\mathrm{CN}_{\mathrm{str}}\right) ;{ }^{1} \mathrm{H} \mathrm{NMR}\left(\mathrm{CDCl}_{3}\right) \delta 2.41\left(\mathrm{~s}, 3 \mathrm{H}, \mathrm{CH}_{3}\right), 3.13-3.36(\mathrm{dd}$, $\left.1 \mathrm{H}, \mathrm{H}_{\mathrm{A}}, J_{\mathrm{AB}}=4.6 \mathrm{~Hz}, J_{\mathrm{AX}}=4.6 \mathrm{~Hz}\right), 3.63-3.84\left(\mathrm{dd}, 1 \mathrm{H}, \mathrm{H}_{\mathrm{B}}\right.$, $\left.J_{\mathrm{AB}}=11.2 \mathrm{~Hz}, J_{\mathrm{BX}}=11.2 \mathrm{~Hz}\right), 5.56-5.67\left(\mathrm{dd}, 1 \mathrm{H}, \mathrm{H}_{\mathrm{X}}, J_{\mathrm{AX}}=\right.$ $\left.4.6 \mathrm{~Hz}, J_{\mathrm{BX}}=4.6 \mathrm{~Hz}\right), 6.66\left(\mathrm{~d}, 2 \mathrm{H}, \mathrm{NH}_{2}, J=8.6 \mathrm{~Hz}, \mathrm{D}_{2} \mathrm{O}\right.$ exchangeable), 7.21-7.84 (m, 14H, ArH).

2-Amino-4-(4-methoxyphenyl)-6-[3-(4-nitrophenyl)-5-phenyl-4,5-dihydropyrazol-1-yl]nicotinonitrile (3s): Yield 73\%, $\mathrm{mp} 248^{\circ} \mathrm{C}$. IR $(\mathrm{KBr}) \mathrm{cm}^{-1}: 3413\left(\mathrm{NH}_{2}\right), 2959\left(\mathrm{Ar}-\mathrm{CH}_{\mathrm{str}}\right), 1245$ $\left(\mathrm{CN}_{\mathrm{str}}\right) ;{ }^{1} \mathrm{H} \mathrm{NMR}\left(\mathrm{CDCl}_{3}\right) \delta 3.58\left(\mathrm{~s}, 3 \mathrm{H}, \mathrm{OCH}_{3}\right), 3.14-3.29(\mathrm{dd}$, $\left.1 \mathrm{H}, \mathrm{H}_{\mathrm{A}}, J_{\mathrm{AB}}=4.6 \mathrm{~Hz}, J_{\mathrm{AX}}=4.6 \mathrm{~Hz}\right), 3.61-3.74\left(\mathrm{dd}, 1 \mathrm{H}, \mathrm{H}_{\mathrm{B}}\right.$, $\left.J_{\mathrm{AB}}=11.2 \mathrm{~Hz}, J_{\mathrm{BX}}=11.2 \mathrm{~Hz}\right), 5.59-5.71\left(\mathrm{dd}, 1 \mathrm{H}, \mathrm{H}_{\mathrm{X}}, J_{\mathrm{AX}}=\right.$ $\left.4.6 \mathrm{~Hz}, J_{\mathrm{BX}}=4.6 \mathrm{~Hz}\right), 6.67\left(\mathrm{~d}, 2 \mathrm{H}, \mathrm{NH}_{2}, J=8.8 \mathrm{~Hz}, \mathrm{D}_{2} \mathrm{O}\right.$ exchangeable), 7.16-7.79 (m, 14H, ArH).

2-Amino-6-[3-(4-nitrophenyl)-5-phenyl-4,5-dihydropyrazol-1-yl]-4-pheny Inicotinonitrile (3t): Yield 66\%, mp $249{ }^{\circ} \mathrm{C}$. IR $(\mathrm{KBr}) \mathrm{cm}^{-1}: 3416\left(\mathrm{NH}_{2}\right), 3008\left(\mathrm{Ar}-\mathrm{CH}_{\mathrm{str}}\right), 1259\left(\mathrm{CN}_{\mathrm{str}}\right) ;{ }^{1} \mathrm{H}$ $\operatorname{NMR}\left(\mathrm{CDCl}_{3}\right) \delta 3.12-3.29\left(\mathrm{dd}, 1 \mathrm{H}, \mathrm{H}_{\mathrm{A}}, J_{\mathrm{AB}}=4.8 \mathrm{~Hz}, J_{\mathrm{AX}}=4.8\right.$ $\mathrm{Hz}), 3.66-3.79\left(\mathrm{dd}, 1 \mathrm{H}, \mathrm{H}_{\mathrm{B}}, J_{\mathrm{AB}}=11.4 \mathrm{~Hz}, J_{\mathrm{BX}}=11.4 \mathrm{~Hz}\right)$, $5.51-5.68\left(\mathrm{dd}, 1 \mathrm{H}, \mathrm{H}_{\mathrm{X}}, J_{\mathrm{AX}}=4.8 \mathrm{~Hz}, J_{\mathrm{BX}}=4.8 \mathrm{~Hz}\right), 6.63(\mathrm{~d}$, $2 \mathrm{H}, \mathrm{NH}_{2}, J=8.4 \mathrm{~Hz}, \mathrm{D}_{2} \mathrm{O}$ exchangeable), 7.14-7.82 (m, $15 \mathrm{H}$, ArH).

\section{Pharmacology}

The investigations were conducted on albino mice of either sex (25 - $30 \mathrm{~g})$. The animals were kept under standard conditions at an ambient temperature of $25 \pm 2{ }^{\circ} \mathrm{C}$ and allowed free access to food and water except at the time they were brought out of the cage. All the experimental protocols were carried out with the permission from Institutional Animal Ethics committee (IAEC), form no. 502. Animals were obtained from Central Animal House Facility, Hamdard University, New Delhi-62. Registration no. and date of registration is 173/CPCSEA, 28 Jan., 2000.

\section{Anticonvulsant Activity.}

Maximal Electroshock Test (MES): The maximal electroshock seizure test was carried out according to the standard protocol. ${ }^{10}$ Albino mice were stimulated through corneal electrodes to $50 \mathrm{~mA}$ current at a pulse of $60 \mathrm{~Hz}$ applied for $0.25 \mathrm{~s}$. Animals were previously administered with the test drug i.p. Abolition of hind limb tonic extension spasm was recorded as the anticonvulsant activity. The test compounds were dissolved in polyethylene glycol (PEG). In the preliminary screening, each compound was administered as an i.p. injection at three dose levels $(30,100$ and $300 \mathrm{mg} / \mathrm{kg}$ body mass) and the anticonvulsant activity was assessed after $0.5 \mathrm{~h}$ and $4.0 \mathrm{~h}$ intervals of administration.

Subcutaneous Pentylenetetrazole Induced Seizure Test (scPTZ): The subcutaneous pentylenetetrazole test was performed according to the known protocol. ${ }^{11}$ This method utilizes pentylenetetrazole $(75 \mathrm{mg} / \mathrm{kg})$ that produces seizures in $>95 \%$ of animals as a $0.5 \%$ solution subcutaneously in the posterior midline. The animals were observed for $30 \mathrm{~min}$. Failure to observe even a threshold seizure (a single episode of clonic spasms of at least $5 \mathrm{~s}$ duration) was defined as protection.

The pharmacological parameters estimated in phase I screening was quantified in phase II screening. Anticonvulsant activity was expressed in terms of the median effective dose $\left(\mathrm{ED}_{50}\right)$. For the determination of $\mathrm{ED}_{50}$ values, groups of 10 mice were given a range of intraperitoneal doses of the test drug until at least three points were established in the range of $10-90 \%$ seizure protection. From the plot of these data, the respective 
$\mathrm{ED}_{50}$ values, $95 \%$ confidence intervals, slope of the regression line, and the standard error of the slope were calculated by means of the computer program by Litchfield and Wilcoxon's method. $^{12}$

Neurotoxicity Screening (NT). The minimal motor impairment was measured in mice by the rotorod test. The mice were trained to stay on an accelerating rotorod of diameter $3.2 \mathrm{~cm}$ that rotates at $10 \mathrm{rpm}$. Trained animals were given i.p. injection of the test compounds 30,100 and $300 \mathrm{mg} / \mathrm{kg}$. Neurotoxicity was indicated by the inability of the animal to maintain equilibrium on the rod for at least $1 \mathrm{~min}$. in each of the trials. ${ }^{13}$

Acute Toxicity Studies. The most active compounds were tested for their acute toxic effects on mice. In this test, the animals were administered a range of intraperitoneal doses of compounds and the toxic effects, if any, were studied.

Liver Enzymes Estimation. The biochemical parameters such as serum glutamate oxaloacetate transaminase (SGOT) and serum glutamate pyruvate transaminase (SGPT) were estimated by Rietman and Frankel's method ${ }^{14}$ and alkaline phosphatase was measured by using King and Armstrong method. ${ }^{15}$

Statistical Analyses. All the statistical analyses were performed using software GraphPad InStat 3.0 version and the method employed was ANOVA followed by dunnett's multiple comparison test.

In silico Studies.

Distance Mapping: In the conformational analysis of the five known clinically active anticonvulsant drugs that act according to the same mechanism by blockade of voltage-dependent sodium channels such as phenytoin, carbamazepine, lamotrigine, rufinamide and zonisamide, a molecular model was suggested on the basis of molecular dynamics distance estimations. ${ }^{9}$ According to the model the distances between the three essential pharmacophoric elements should be optimum and within the suggested range for good anticonvulsant activity. For the molecular mechanics calculations of compounds 3a-t, the CS Chem3D Pro 6.0 version program was used for employing the CHARMM force field. ${ }^{16}$

Three-dimensional Structural Analyses. This was performed to delineate the role of three-dimensional structures of synthesized compounds to their anticonvulsant activity. Compound 2-Amino-6-(3,5-diphenyl-4,5-dihydropyrazol-1-yl)-4phenylnicotinonitrile (3e) which represents the prototype structure of the synthesized compounds was chosen for the study. It was performed using the software Ortep3v2.

\section{Results and Discussion}

Chemistry. The synthesis of titled compounds (3a-t) was carried out according to Scheme 1. 4-substituted acetophenones were treated with benzaldehyde in basic condition to yield the corresponding chalcones (1a-d) that in turn refluxed with hydrazine hydrate in acetic acid to afford (2a-d). In the final step, the free acetyl group was cyclized to substituted pyridyl moiety by reacting with substituted aryl aldehydes in presence of malononitrile and ammonium acetate. The physicochemical data are presented in Table 1.

All the final compounds were characterized by different spectral analytical techniques. The ${ }^{1} \mathrm{H}$ NMR spectra showed

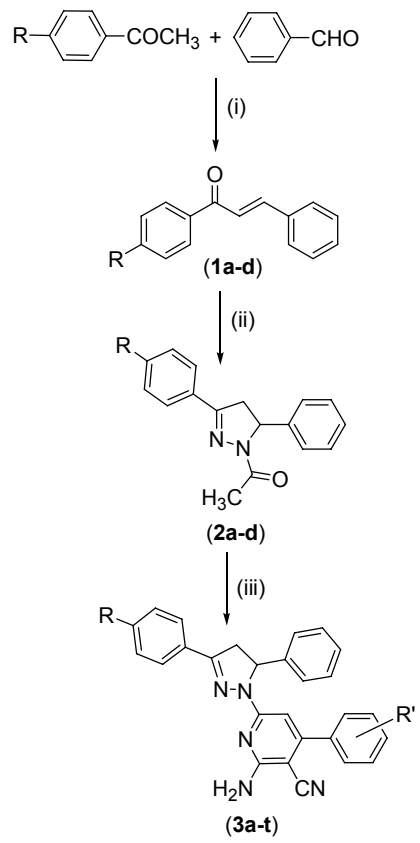

Scheme 1. Reagents and Conditiions: (i) $40 \% \mathrm{NaOH}$, ethanol, $5{ }^{\circ} \mathrm{C}$; (ii) $\mathrm{NH}_{2} \mathrm{NH}_{2} \cdot \mathrm{H}_{2} \mathrm{O}, \mathrm{AcOH}$, reflux; (iii) $\mathrm{R}^{\prime} \mathrm{PhCHO}$, malononitrile, ammonium acetate, ethanol, reflux

characteristic pattern of pyrazoline moiety with three distinct double-doublets of the three protons coupling with each other. The two protons of the amino group were observed at $6.1-6.69$ ppm as a doublet. The multiplet in the range of $7.11-7.89 \mathrm{ppm}$ was assigned for the aromatic protons. The hydroxyl group attached to the aromatic ring showed a singlet in the range of 9.31 9.51 that was $\mathrm{D}_{2} \mathrm{O}$ exchangeable. The IR spectra corresponded to the structure of the compounds and showed presence of sharp and intense peaks of different functional groups present. The band for hydroxyl group was present in the range of 3615 $3715 \mathrm{~cm}^{-1}$ whereas a sharp band in the range of $3412-3478$ $\mathrm{cm}^{-1}$ was of primary amino group. The aromatic C-H stretching was observed in the range of $2896-3064 \mathrm{~cm}^{-1}$ and the C-N stretching of the nitrile group at $1178-1289 \mathrm{~cm}^{-1}$.

Pharmacology.

Anticonvulsant Activity: The anticonvulsant activity was assessed by two most adopted animal models electroshock (MES) and chemoshock (scPTZ) methods. All the synthesized compounds were administered intraperitoneally into mice using doses of 30,100 and $300 \mathrm{mg} / \mathrm{kg}$ and the observations were taken at two different time intervals $(0.5 \mathrm{~h}$ and $4.0 \mathrm{~h})$. Neurological impairment was evaluated by rotorod method and the data are presented in Table 2.

In the phase I preliminary anticonvulsant screening, all the compounds showed protection in MES screen which was the indicative of the good ability of these compounds to prevent the seizure spread. Among these compounds, 3f, 3i, 3p and 3s showed protection from seizure at the lowest dose $30 \mathrm{mg} / \mathrm{kg}$ after $0.5 \mathrm{~h}$. Interestingly, $\mathbf{3 i}$ and $\mathbf{3 s}$ continued the anticonvulsant activity after $4.0 \mathrm{~h}$ at the same dose. Compounds $\mathbf{3 f}$ and $\mathbf{3 p}$ also displayed protection after $4.0 \mathrm{~h}$ but at $100 \mathrm{mg} / \mathrm{kg}$ dose, which indicated the promising nature of the compounds showing quick onset and long duration of action at relatively lower doses. Com- 
Table 1. Physicochemical parameters of the titled compounds (3a-t)

\begin{tabular}{|c|c|c|c|c|c|c|c|c|}
\hline \multirow{2}{*}{ Compound } & \multirow{2}{*}{$\mathrm{R}$} & \multirow{2}{*}{$\mathrm{R}^{\prime}$} & \multirow{2}{*}{ Mol. Formula $^{a}$ (Mol. wt.) } & \multirow{2}{*}{$C \log \mathrm{P}^{b}$} & \multirow{2}{*}{$R_{f}^{c}$ value } & \multicolumn{3}{|c|}{ Elemental analyses \% found (calculated) } \\
\hline & & & & & & $\mathrm{C}$ & $\mathrm{H}$ & $\mathrm{N}$ \\
\hline $3 \mathbf{a}$ & $\mathrm{H}$ & $4-\mathrm{OH}$ & $\mathrm{C}_{27} \mathrm{H}_{21} \mathrm{~N}_{5} \mathrm{O}(431.49)$ & 5.40 & 0.59 & $75.09(75.16)$ & $4.85(4.91)$ & $16.21(16.23)$ \\
\hline $\mathbf{3 b}$ & $\mathrm{H}$ & $2-\mathrm{OH}$ & $\mathrm{C}_{27} \mathrm{H}_{21} \mathrm{~N}_{5} \mathrm{O}(431.49)$ & 4.90 & 0.64 & $75.12(75.16)$ & $4.94(4.91)$ & $16.19(16.23)$ \\
\hline $3 \mathbf{c}$ & $\mathrm{H}$ & $4-\mathrm{CH}_{3}$ & $\mathrm{C}_{28} \mathrm{H}_{23} \mathrm{~N}_{5}(429.52)$ & 6.3 & 0.75 & $78.26(78.30)$ & $5.41(5.40)$ & $16.32(16.31)$ \\
\hline 3d & $\mathrm{H}$ & $4-\mathrm{OCH}_{3}$ & $\mathrm{C}_{28} \mathrm{H}_{23} \mathrm{~N}_{5} \mathrm{O}(445.52)$ & 5.8 & 0.84 & $75.45(75.49)$ & $5.22(5.20)$ & $15.75(15.72)$ \\
\hline $3 e$ & $\mathrm{H}$ & $\mathrm{H}$ & $\mathrm{C}_{27} \mathrm{H}_{21} \mathrm{~N}_{5}(415.49)$ & 5.87 & 0.51 & $78.01(78.05)$ & $5.11(5.09)$ & $16.89(16.86)$ \\
\hline 3f & $\mathrm{Br}$ & $4-\mathrm{OH}$ & $\mathrm{C}_{27} \mathrm{H}_{20} \mathrm{BrN}_{5} \mathrm{O}(510.38)$ & 6.26 & 0.63 & $63.57(63.54)$ & $3.98(3.95)$ & $13.73(13.72)$ \\
\hline $3 g$ & $\mathrm{Br}$ & $2-\mathrm{OH}$ & $\mathrm{C}_{27} \mathrm{H}_{20} \mathrm{BrN}_{5} \mathrm{O}(510.38)$ & 5.76 & 0.69 & $63.58(63.54)$ & $3.96(3.95)$ & $13.74(13.72)$ \\
\hline $3 \mathbf{h}$ & $\mathrm{Br}$ & $4-\mathrm{CH}_{3}$ & $\mathrm{C}_{28} \mathrm{H}_{22} \mathrm{BrN}_{5}(508.41)$ & 7.23 & 0.77 & $66.21(66.15)$ & $4.35(4.36)$ & $13.75(13.77)$ \\
\hline $3 \mathbf{i}$ & $\mathrm{Br}$ & $4-\mathrm{OCH}_{3}$ & $\mathrm{C}_{28} \mathrm{H}_{22} \mathrm{BrN}_{5} \mathrm{O}(524.41)$ & 6.75 & 0.86 & $64.14(64.13)$ & $4.20(4.23)$ & $13.32(13.35)$ \\
\hline $3 \mathbf{j}$ & $\mathrm{Br}$ & $\mathrm{H}$ & $\mathrm{C}_{27} \mathrm{H}_{20} \mathrm{BrN}_{5}(494.39)$ & 6.73 & 0.60 & $65.53(65.59)$ & $4.11(4.08)$ & $14.15(14.17)$ \\
\hline $3 \mathbf{k}$ & $\mathrm{Cl}$ & $4-\mathrm{OH}$ & $\mathrm{C}_{27} \mathrm{H}_{20} \mathrm{ClN}_{5} \mathrm{O}(465.93)$ & 6.11 & 0.59 & $69.64(69.60)$ & $4.37(4.33)$ & $15.08(15.03)$ \\
\hline 31 & $\mathrm{Cl}$ & $2-\mathrm{OH}$ & $\mathrm{C}_{27} \mathrm{H}_{20} \mathrm{ClN}_{5} \mathrm{O}(465.93)$ & 5.61 & 0.63 & $69.64(69.60)$ & $4.31(4.33)$ & $15.05(15.03)$ \\
\hline $3 \mathrm{~m}$ & $\mathrm{Cl}$ & $4-\mathrm{CH}_{3}$ & $\mathrm{C}_{28} \mathrm{H}_{22} \mathrm{ClN}_{5}(463.96)$ & 7.08 & 0.74 & $72.43(72.48)$ & $4.75(4.78)$ & $15.13(15.09)$ \\
\hline $3 n$ & $\mathrm{Cl}$ & $4-\mathrm{OCH}_{3}$ & $\mathrm{C}_{28} \mathrm{H}_{22} \mathrm{ClN}_{5} \mathrm{O}(479.96)$ & 6.60 & 0.78 & $70.02(70.07)$ & $4.65(4.62)$ & $14.54(14.59)$ \\
\hline 30 & $\mathrm{Cl}$ & $\mathrm{H}$ & $\mathrm{C}_{27} \mathrm{H}_{20} \mathrm{ClN}_{5}(449.93)$ & 6.58 & 0.55 & $72.12(72.07)$ & $4.46(4.48)$ & $15.59(15.57)$ \\
\hline $3 \mathbf{p}$ & $\mathrm{NO}_{2}$ & $4-\mathrm{OH}$ & $\mathrm{C}_{27} \mathrm{H}_{20} \mathrm{~N}_{6} \mathrm{O}_{3}(476.49)$ & 5.14 & 0.59 & $68.07(68.06)$ & $4.21(4.23)$ & $17.69(17.64)$ \\
\hline $3 q$ & $\mathrm{NO}_{2}$ & $2-\mathrm{OH}$ & $\mathrm{C}_{27} \mathrm{H}_{20} \mathrm{~N}_{6} \mathrm{O}_{3}(476.49)$ & 4.64 & 0.64 & $68.07(68.06)$ & $4.26(4.23)$ & $17.68(17.64)$ \\
\hline $3 r$ & $\mathrm{NO}_{2}$ & $4-\mathrm{CH}_{3}$ & $\mathrm{C}_{28} \mathrm{H}_{22} \mathrm{~N}_{6} \mathrm{O}_{2}(474.51)$ & 6.11 & 0.75 & $70.84(70.87)$ & $4.68(4.67)$ & $17.70(17.71)$ \\
\hline $3 s$ & $\mathrm{NO}_{2}$ & $4-\mathrm{OCH}_{3}$ & $\mathrm{C}_{28} \mathrm{H}_{22} \mathrm{~N}_{6} \mathrm{O}_{3}(490.51)$ & 5.63 & 0.79 & $68.59(68.56)$ & $4.50(4.52)$ & $17.16(17.13)$ \\
\hline $3 t$ & $\mathrm{NO}_{2}$ & $\mathrm{H}$ & $\mathrm{C}_{27} \mathrm{H}_{20} \mathrm{~N}_{6} \mathrm{O}_{2}(460.49)$ & 5.61 & 0.61 & $70.48(70.42)$ & $4.36(4.38)$ & $18.26(18.25)$ \\
\hline
\end{tabular}

${ }^{a}$ Solvent of crystallization-Ethanol. ${ }^{b}$ CLog P was calculated using software ACD Labs Software 2.0. ${ }^{c}$ Solvent system - Toluene:Ethyl acetate:Formic acid $(5: 4: 1)$.

Table 2. Anticonvulsant activity and minimal motor impairment data of the synthesized compounds (3a-t)

\begin{tabular}{|c|c|c|c|c|c|c|}
\hline \multirow{3}{*}{ Compound } & \multicolumn{6}{|c|}{ Intraperitoneal injection in mice $^{a}$} \\
\hline & \multicolumn{2}{|c|}{ MES screen } & \multicolumn{2}{|c|}{ scPTZ screen } & \multicolumn{2}{|c|}{$\begin{array}{l}\text { Neurotoxicity } \\
\text { screen }\end{array}$} \\
\hline & $0.5 \mathrm{~h}$ & $4.0 \mathrm{~h}$ & $0.5 \mathrm{~h}$ & $4.0 \mathrm{~h}$ & $0.5 \mathrm{~h}$ & $4.0 \mathrm{~h}$ \\
\hline $3 \mathbf{a}$ & 100 & 100 & $-b$ & 300 & - & - \\
\hline $3 \mathbf{b}$ & 300 & 300 & - & - & - & - \\
\hline $3 \mathbf{c}$ & - & 300 & - & 300 & - & 300 \\
\hline 3d & 100 & 300 & 100 & 300 & - & - \\
\hline $3 \mathbf{e}$ & - & 300 & - & - & - & 300 \\
\hline $3 f$ & 30 & 100 & 300 & 300 & 300 & - \\
\hline $3 g$ & 100 & 100 & - & 300 & 300 & 300 \\
\hline $3 \mathbf{h}$ & 100 & 300 & - & - & 100 & 300 \\
\hline $3 \mathbf{i}$ & 30 & 30 & 100 & - & - & - \\
\hline $3 \mathbf{j}$ & 300 & - & - & - & 300 & 300 \\
\hline $3 \mathbf{k}$ & 100 & 300 & 100 & 100 & 300 & 300 \\
\hline 31 & 300 & 300 & 300 & - & - & - \\
\hline $3 m$ & - & 300 & - & - & 300 & - \\
\hline $3 n$ & 100 & 300 & 100 & 100 & - & 300 \\
\hline 30 & 300 & - & - & 300 & 300 & - \\
\hline $3 p$ & 30 & 100 & 300 & 300 & 100 & 300 \\
\hline $3 q$ & 300 & 300 & - & 300 & - & 100 \\
\hline $3 r$ & 300 & - & 300 & 300 & 300 & 100 \\
\hline $3 s$ & 30 & 30 & 100 & 300 & - & - \\
\hline $3 t$ & 300 & - & - & 300 & - & 300 \\
\hline Phenytoin & 30 & 30 & - & - & 100 & 100 \\
\hline Ethosuximide & - & - & 100 & 300 & - & - \\
\hline Phenobarbital & 100 & 30 & - & - & 100 & 300 \\
\hline
\end{tabular}

${ }^{a}$ Number of animals used $=6$; Solvent used- Polyethylene glycol; Dose of 30,100 and $300 \mathrm{mg} / \mathrm{kg}$ were administered i.p. The figures in the table indicate the minimum dose whereby bioactivity was demonstrated in half or more of the mice. The animals were examined at $0.5 \mathrm{~h}$ and $4 \mathrm{~h}$ after injections were administered. ${ }^{b}$ The dash $(-)$ indicates an absence of activity at maximum dose administered $(300 \mathrm{mg} / \mathrm{kg})$. pounds $3 \mathbf{a}, \mathbf{3 d}, \mathbf{3 g}, \mathbf{3 h}, \mathbf{3 k}$ and $\mathbf{3 n}$ were found to be active in the MES screen after $0.5 \mathrm{~h}$ at a dose of $100 \mathrm{mg} / \mathrm{kg}$. Among these compounds, $\mathbf{3} \mathbf{a}$ and $\mathbf{3} \mathbf{g}$ continued to protect from seizures at the same dose after $4.0 \mathrm{~h}$. Rest all the compounds showed the activity at the higher dose of $300 \mathrm{mg} / \mathrm{kg}$.

In the scPTZ screen, compounds that were found to be active included 3a, 3c, 3d, 3f, 3g, 3i, 3k, 3l, 3n, 3o, 3p, 3q, 3r, 3s and 3t. Among these compounds, 3d, 3i, 3k, 3n and $\mathbf{3 s}$ were active at $100 \mathrm{mg} / \mathrm{kg}$ after $0.5 \mathrm{~h}$. Compound $\mathbf{3 k}$ and $\mathbf{3 n}$ showed the promising nature by continuing the protection from the chemically induced seizures at the same dose after $4.0 \mathrm{~h}$. Compounds $\mathbf{3 d}$ and $3 \mathrm{~s}$ were also found to be active after $4.0 \mathrm{~h}$ but at a higher dose of $300 \mathrm{mg} / \mathrm{kg}$. Compounds that were active at $300 \mathrm{mg} / \mathrm{kg}$ dose after $0.5 \mathrm{~h}$ were $3 f, 3 \mathrm{l}, \mathbf{3 p}$ and $3 \mathrm{r}$. Compounds $3 \mathbf{3 f}, \mathbf{3 p}$ and $3 \mathrm{r}$ were also active at the same dose after $4.0 \mathrm{~h}$.

In the neurotoxicity screening, compounds that were devoid of minimal motor impairment at any dose were $\mathbf{3 a}, \mathbf{3 b}, \mathbf{3} \mathbf{d}, \mathbf{3 i}$, $3 \mathbf{l}$ and 3s. Rest all the compounds showed some sign of neurotoxicity but none of the compounds showed neurotoxicity pattern like phenytoin does.

In the phase II anticonvulsant screening, the most active compounds $\mathbf{3 i}$ and $\mathbf{3 s}$ were quantitatively evaluated for their anticonvulsant activity $\left(\mathrm{ED}_{50}\right)$ and the results are presented in Table 3. Both the compounds showed comparable anticonvulsant activity to the standard drugs. Compound $\mathbf{3 i}$ displayed good anti-MES activity with $\mathrm{ED}_{50}$ of 17.5 which was slightly lesser to the currently used antiepileptic drugs phenytoin and carbamazepine and better than that of phenobarbital and valproate. It also showed encouraging activity in the chemoshock screen with $\mathrm{ED}_{50}$ value of 154.1 indicating the versatile nature of the compounds showing good activity in more than one type of seizures. Nevertheless, compound 3s was also found to have 
Table 3. Phase II quantitative anticonvulsant evaluation in mice

\begin{tabular}{ccc}
\hline \multirow{2}{*}{ Compound } & \multicolumn{2}{c}{ ED $_{50}{ }^{a}$} \\
\cline { 2 - 3 } & MES & scPTZ \\
\hline 3i & $17.5(14.2-21.4)^{b}$ & $154.1(136.7-174.4)$ \\
3s & $22.6(17.1-26.4)$ & $242.6(226.4-263.4)$ \\
Phenytoin & $9.5(8.1-10.4)$ & $>300$ \\
Carbamazepine & $8.8(5.5-14.1)$ & $>100$ \\
Phenobarbital & $21.8(21.8-25.5)$ & $13.2(5.8-15.9)$ \\
Valproate & $272(247-338)$ & $149(123-177)$ \\
\hline
\end{tabular}

Number of animals used $=10$; Solvent used: polyethylene glycol $(0.1 \mathrm{~mL}$, i.p.). ${ }^{a}$ Dose in milligrams per kilogram body mass. ${ }^{b}$ Data in parentheses are the $95 \%$ confidence limits.

considerable anticonvulsant activity in MES screen having $\mathrm{ED}_{50}$ value 22.6 and in scPTZ screen having $\mathrm{ED}_{50}$ value 242.6.

Designing of the compounds were made to study the structure activity relationship of the series as the phenyl ring attached to the pyrazoline moiety was either kept unsubstituted or substituted at fourth position by electron withdrawing groups. The other phenyl ring that was attached to the pyridyl moiety was either substituted with the electron releasing groups at different positions or kept unsubstituted. Substitutions at the phenyl ring attached to the pyrazoline moiety had a marked effect on the anti-MES activity since the size of the electron-withdrawing group attached played a major role in determining it. Substitutions with larger $\mathrm{NO}_{2}$ and $\mathrm{Br}$ groups resulted in an increase in the activity than the smaller $\mathrm{Cl}$ and $\mathrm{H}$ atoms. On the other hand, substitutions at the fourth position in the phenyl ring attached to the pyridyl moiety were more favorable than the other positions. 4-Hydroxy and 4-methoxy derivatives were the most potent of the series and substitutions with other groups resulted in the decrease in activity. In the chemoshock screen the chloro substituted derivatives were more effective of the series followed by nitro derivatives. The other substitutions did not seem to have any marked effect on the activity. The bromo-substituted derivatives were found to be comparatively more neurotoxic than the other compounds.

Acute Toxicity Studies. The acute toxicity studies were performed on the albino mice in order to assess any mortality or toxic effects shown by the synthesized compounds at increased doses and the data are presented in Table 4 . The most active compounds were dissolved in PEG-400 and different doses were administered intraperitoneally to mice and the behavioral changes are seen and compared with phenytoin, carbamazepine and phenobarbital. Unlike the standard drugs, it was found that both the tested compounds did not produce any mortality or gross effect on the central nervous system at any of the dose levels tested, i.e., 5, 25, 125, 250 and $400 \mathrm{mg} / \mathrm{kg}$. However, the animals have exhibited some of the toxic signs such as chewing, licking, salivation followed by brief period of sedation. There was not any toxicity seen in the vehicle alone as evident in the control group of animals.

Liver Enzymes Estimation. Since some currently used antiepileptic drugs boast liver toxicity as one of the restraint, the liver enzyme estimation of the most active compounds $\mathbf{3} \mathbf{i}$ and 3s were also performed to check the enormity of liver toxicity
Table 4. Acute toxicity study data of selected compounds

\begin{tabular}{cccccc}
\hline & \multicolumn{5}{c}{$\begin{array}{c}\text { Number of animals dead/ } \\
\text { Total number of animals tested }\end{array}$} \\
\cline { 2 - 6 } Compounds & \multicolumn{5}{c}{ Dose (mg/kg), i.p. } \\
\cline { 2 - 6 } & 5 & 25 & 125 & 250 & 400 \\
\hline Control & $0 / 6$ & $0 / 6$ & $0 / 6$ & $0 / 6$ & $0 / 6$ \\
3i & $0 / 6$ & $0 / 6$ & $0 / 6$ & $0 / 6$ & $0 / 6$ \\
3s & $0 / 6$ & $0 / 6$ & $0 / 6$ & $0 / 6$ & $0 / 6$ \\
Phenytoin & $0 / 6$ & $0 / 6$ & $0 / 6$ & $3 / 6$ & $4 / 6$ \\
Carbamazepine & $0 / 6$ & $0 / 6$ & $0 / 6$ & $3 / 6$ & $3 / 6$ \\
Phenobarbital & $0 / 6$ & $0 / 6$ & $0 / 6$ & $3 / 6$ & $3 / 6$ \\
\hline
\end{tabular}

Vehicle used: Polyethylene glycol ( $0.4 \mathrm{~mL}$, i.p.)

Table 5. Liver enzymes estimation of selected compounds

\begin{tabular}{cccc}
\hline Treatment & $\begin{array}{c}\text { Alkaline phosphatase } \\
(\text { Mean } \pm \text { SEM) }\end{array}$ & $\begin{array}{c}\text { SGOT level } \\
(\text { Mean } \pm \text { SEM) }\end{array}$ & $\begin{array}{c}\text { SGPT level } \\
(\text { Mean } \pm \text { SEM })\end{array}$ \\
\hline Control & $17.84 \pm 0.612$ & $134.23 \pm 3.256$ & $41.83 \pm 2.312$ \\
3i & $18.92 \pm 0.835$ & $128.43 \pm 2.817$ & $39.12 \pm 2.729$ \\
3s & $19.14 \pm 1.134$ & $144.48 \pm 3.476 *$ & $38.15 \pm 3.342$ \\
\hline
\end{tabular}

$* \mathrm{P}<0.05$; The mean levels of enzymes were calculated using ANOVA followed by Dunnett's multiple comparison test.

and the data are presented in Table 5. The concentrations of alkaline phosphatase, SGOT and, SGPT were determined in serum and the values are represented as mean \pm SEM. Compound $3 \mathbf{i}$ showed no any significant change in enzymes level as compared to the control. Compound $3 \mathrm{~s}$ was observed to increase the SGOT level moderately $(\mathrm{P}<0.05)$ but the other values were not significant indicating the non-toxic nature of the tested compounds.

\section{In silico Studies.}

Distance Mapping: Comparison of the structures of the synthesized compounds and other molecules with anticonvulsant activity were performed to find out the structural elements essential for action. The compounds selected for this comparison have at least one aryl (R) hydrophobic domain, one electron donor (D) and a hydrogen bond acceptor/donor unit (HAD). In an initial study, calculations on the basis of molecular mechanics, with the force field based on CHARMM parameterization were performed to obtain an overview on their minimum conformation for bioactivity. The synthesized compounds were examined to check whether they reflect the conditions of the derived pharmacophore model. Analyses of the distance relationship showed that synthesized compounds (3a-t) fulfil the essential demands of pharmacophore when compared with other known anticonvulsant drugs. In case of the titled compounds

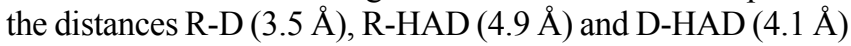
were in conformity with the distances of active anticonvulsant drugs that should be in the range of R-D (3.2 - 5.1 $\AA$ ), R-HAD (4.2 - $8.5 \AA)$ and D-HAD (3.9 - $5.5 \AA)$.

Three-dimensional Structural Analyses. The ORTEP diagram $(50 \%$ probability) was drawn using the software Ortep3v2 and the characteristics were studied. As noted in Fig. 2 of the compound $3 \mathbf{e}$, strong hydrogen bondings occurred between the vinyl protons and the nitrogen atoms. A total of four potential 


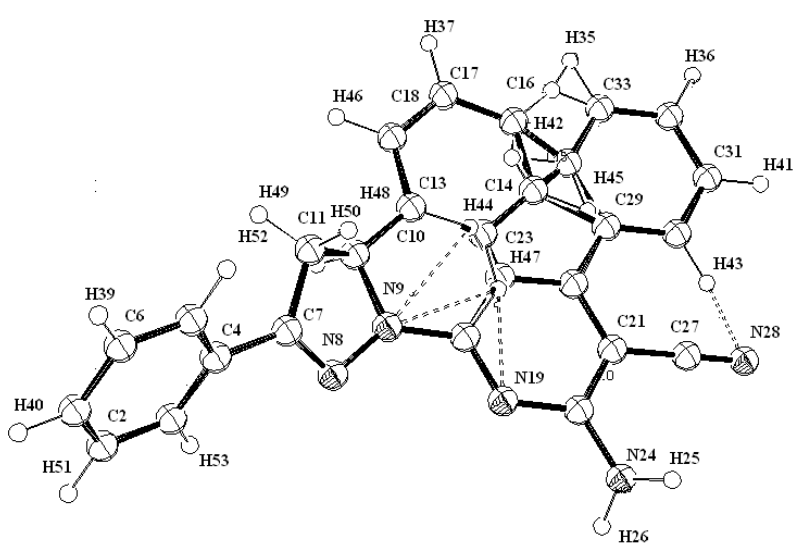

Figure 2. ORTEP diagram (50\% probability) of 2-Amino-6-(3,5-diphenyl-4,5-dihydropyrazol-1-yl)-4-phenylnicotinonitrile (3e) showing four hydrogen bonding interactions $(\mathrm{N} \cdots \mathrm{H})$ as dotted line.

hydrogen bonds were found. First found between N9 and H44 where the distance between the donor and the acceptor atom $\mathrm{d}(\mathrm{N} \cdots \mathrm{H})$ was found to be $2.657 \AA$. Second was observed between $\mathrm{N} 9$ and $\mathrm{H} 47$ where $(\mathrm{N} \cdots \mathrm{H})$ distance was found to be $2.311 \AA$. Third hydrogen bond was observed between N19 and $\mathrm{H} 47$ where $(\mathrm{N} \cdots \mathrm{H})$ distance was calculated to be $2.172 \AA$.

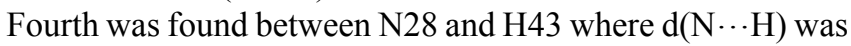
found to be $1.644 \AA$. These hydrogen bondings ensure the rings are planar. It was reported that the planarity of molecules is an essential factor in determination of biological activity. ${ }^{17}$

\section{Conclusions and Future Directions}

In conclusion, the rationally designed pyrazolo-pyridines can be regarded as a new structural class of anticonvulsants that have shown comparable anticonvulsant activity to the some standard drugs with markedly lower neurotoxicity and hepatotoxicity. These can act as lead molecules for future investigations and need to be explored further to get better agents.
Acknowledgments. One of the authors (W.A.) is thankful to Council of Scientific and Industrial Research (CSIR), Government of India for the Senior Research Fellowship (SRF) as financial assistance. We are also thankful to Shrenik Pharma Ltd., Mumbai, India for providing pentylenetetrazole as gift sample.

\section{References}

1. Ozdemir, Z.; Kandilci, H. B.; Gümüşel, B.; Caliş, U.; Bilgin, A. A. Eur. J. Med. Chem. 2007, 42, 373-379.

2. Ozdemir, Z.; Burak, K. H.; Gumusel, B.; Calis, U.; Altan, B. A. Arch. Pharm. 2008, 341, 701-707.

3. Abdel-Aziz, M.; El-Din, A. G.; Hassan, A. A. Eur. J. Med. Chem. 2001, 44, 3480-3487.

4. Lukevits, É. Chem. Heter. Comp. 1995, 31, 639-650.

5. Narendar, P.; Parthiban, J.; Anbalagan, N.; Gunasekaran, V.; Leonard, T. J. Biol. Pharm. Bull. 2003, 26, 182-187.

6. Bin, H.; Crider, A. M.; Stables, J. P. Eur. J. Med. Chem. 2001, 36, 265-286.

7. Kaminski, K.; Obniska, J.; Zagorska, A.; Maciag, D. Arch. Pharm. 2006, 339, 255-261.

8. Siddiqui, N.; Alam, P.; Ahsan, W. Arch. Pharm. 2009, 342, 173181.

9. Mamolo, M. G.; Zampieri, D.; Falagiani, V.; Vio, L.; Banfi, E. IL Farmaco 2001, 56, 593-599.

10. Krall, R. L.; Penry, J. K.; White, B. G.; Kupferberg, H. J.; Swinyard, E. A. Epilepsia 1978, 19, 409-428.

11. Clark, C. R.; Wells, M. J. M.; Sansom, R. T.; Norris, G. N.; Dockens, R. C.; Ravis, W. R. J. Med. Chem. 1984, 27, 779-782.

12. Litchfield, J. T.; Wilcoxon, F. J. Pharmacol. Exp. Ther. 1949, 96, 99-113.

13. Dunham, N. W.; Miya, T. A. J. Am. Pharm. Assoc. Sci. 1957, 46, 208-209.

14. Reitman, S.; Frankel, S. A. Am. J. Clin. Pathol. 1957, 28, 56-63.

15. King, E. J.; Armstrong, A. R. Can. Med. Assoc. J. 1934, 31, 376381.

16. Brooks, B. R.; Bruccoleri, R. E.; Olafson, B. D.; States, D. J.; Swaminathan, S.; Karplus, M. J. Comput. Chem. 1983, 4, 187-217.

17. Eddington, N. D.; Cox, D. S.; Roberts, R. R.; Butcher, R. J.; Edafiogho, I. O.; Stables, J. P.; Cooke, N.; Goodwin, A. M.; Smith, C. A.; Scott, K. R. Eur. J. Med. Chem. 2002, 37, 635-648. 